\section{Stamp out shabby research conduct}

As heads of funding bodies for medical research, we are concerned that questionable practices among researchers seem to be becoming more prevalent. Although these do not meet current definitions of misconduct, they can still distort biomedical science and cause irreproducibility - with potentially critical consequences for policies and patients.

For example, researchers may cut corners by withholding methodological details or by failing to disclose data for independent scrutiny. Inadequate training can also be responsible for false conclusions arising from flawed experimental design, methodology or statistical analysis. Some countries, including Australia, Canada and the Netherlands, have a category for these - 'poor conduct'. This must be addressed if proved, even though it is less egregious than research misconduct.

International funding bodies, informally convening with heads of international biomedical research organizations, have agreed to undertake a worldwide analysis of definitions of different types of misconduct and the policies used to tackle them. This should help to harmonize standards of research rigour and integrity globally, for the ultimate benefit of patients. Warwick P. Anderson ${ }^{\star}$ National Health and Medical Research Council of Australia, Canberra, Australia. warwick.anderson@nhmrc.gov.au ${ }^{*}$ On behalf of 4 correspondents (see go.nature.com/adxrpe for full list).

\section{Tax transactions to stabilize trading}

An obvious method for controlling high-speed trading (M. Buchanan Nature 518, $161-163 ; 2015)$ is a global financial-transaction tax of the kind proposed by the
European Union in 2011.

Such a tax, originally designed to raise revenue, could be set to lead to a typical trading time. There would be no need to regulate trading times explicitly: it would simply not be profitable to trade on the tiny, rapid fluctuations that now trigger transactions. This solution would be simpler - and, with its revenues, more beneficial - than technical approaches such as 'speed bumps' that delay transactions.

The non-equilibrium, complex systems that correspond to economies often operate at the threshold of instability. Adding taxes ('friction') should not be seen as creating inefficiency, but as a stabilizing influence that can avoid the costs of dramatic crashes.

John Bechhoefer Simon Fraser University, Burnaby, Canada. johnb@sfu.ca

\section{Undergraduate research in action}

Our programmes at California State University address the challenges of bringing undergraduates into research labs (see Nature 518, 127-128; 2015). The students are then better equipped for admission to the top professional training programmes in the United States and worldwide.

More than 100 undergraduate research students are trained every year under our programmes, which have been running for 43 years and have garnered a US Presidential Award for Mentoring, among other honours. We aim to make students proficient in doing quality research experiments and in statistically analysing and publishing them.

All new undergrads are trained by peer undergraduates (not graduate students or postdocs) experienced in the research, ensuring that the newcomers immediately feel comfortable in the research setting; their work is regularly

checked by senior staff.

The burden of heavy course loads is mitigated by an openlab policy that allows students to pursue their research out of hours and during university vacations.

Our undergraduates have co-authored hundreds of publications and national presentations. And, to stimulate pre-college students' interest in research, we established a journal of student research abstracts almost 20 years ago (now open access), and annual symposia for student research posters (see go.nature.com/dwox6d). Steven B. Oppenheimer California State University, Northridge, California, USA. steven.oppenheimer@csun.edu

\section{Women's grants lost in inequality ocean}

Denmark last year launched its YDUN programme, an experimental one-year government research-funding scheme specifically for women. It was branded as sexist and provoked a political squall, so is unlikely to be repeated. Our analysis indicates that the 110 million krone (US\$16 million) allocated to YDUN is roughly the same as the shortfall in Danish grant money won by women compared with men every year over the past 10 years.

The proportion of successful grant applications in 2009-13 to the Danish Council for Independent Research (DFF), which also ran YDUN, was roughly comparable for male and female researchers according to their own analysis ( $14 \%$ and $11 \%$, respectively; see go.nature.com/uryhca (in Danish)).

However, our analysis of DFF data since the council's foundation in 2005 revealed that this 3\% difference in success rates is significant: it corresponds to a male advantage of an average of 104 million krone per year, comparable to the entire YDUN funding allocation for women.

YDUN was a welcome attempt to widen Denmark's talent pool, but managed to level the playing field for only one year, and only for the DFF. Even then, the success rate for YDUN was only $3 \%$ (17 of 553 applicants). This level of competition is much higher than for DFF funding. Even though YDUN funding effectively made up the shortfall within the DFF for 2014, women still had to compete much harder to get it.

Darach Watson, Jens Hjorth Niels Bohr Institute, University of Copenhagen, Denmark. darach@dark-cosmology.dk

\section{Assessing resistance to new antibiotics}

Losee Ling and colleagues detect no bacterial resistance to the new antibiotic molecule teixobactin (L. L. Ling et al. Nature 517, 455-459; 2015), but this could be because the conditions of their test may limit its sensitivity (see J. Ramsayer et al. Evol. Appl. 6, 608-616; 2013). 'Evolutionary rescue' is a more powerful assay for evaluating the probability of resistance to novel antibiotics in large bacterial samples, and therefore for informing decisions about their usage.

Evolutionary rescue assays can distinguish between resistant mutants that are present initially and those that emerge later (H. A. Orr and R. L. Unckless PLoS Genet. 10, e1004551; 2014). This type of assay can also be used to evaluate factors that contribute to the emergence of bacterial resistance, such as 'horizontal' gene transfer from other bacteria or the presence of bacterial 'mutator' strains with vastly increased mutation rates. Michael E. Hochberg Université Montpellier, France. mhochber@univ-montp2.fr Gunther Jansen ChristianAlbrechts-Universität, Kiel, Germany. 